\title{
Spike development inhibition in the ftin mutant is associated with multiple phenotypic characteristics and regulated multiple biological pathways
}

\author{
Yong-sheng Zheng ${ }^{1}$, Jinpeng Zhang ${ }^{1}$, Cheng Liu ${ }^{2}$, Rongzhi Zhang ${ }^{2}$, Xiajie Ji ${ }^{1}$, Han Zhang ${ }^{2}$, \\ Ruyu $\mathrm{Li}^{2}$, and Weihua Liu ${ }^{1}$ \\ ${ }^{1}$ Chinese Academy of Agricultural Sciences \\ ${ }^{2}$ Shandong Academy of Agricultural Sciences
}

April 28, 2020

\begin{abstract}
Spike development of wheat is closely associated with the ability of response to cold stress and inhibited under cold stress in spring. Morphological investigation showed that the ftin gene in $3558 \mathrm{M}$ is associated with multiple phenotypic characteristics, including fewer tillers, delayed floral transition, and the death of shoot apical meristem. In this study, we systematically researched the genetic nature of spike development using ITRAQ, transcriptome sequencing, western blot and RNAi technologies. The results showed that the ftin mutant is cold sensitive and activates the cold acclimation pathway. Multiple defence responses, including the ROS-mediated hypersensitive response, SA-mediated systemic acquired resistance and FLS2-induced pathogen-associated defence response, were activated to respond to normal cold stress and led to the apex death. Meanwhile, the continuous cold acclimation pathway inhibited the SVP-SCO1-LFY flowering pathway and leads to inhibition of spike development. Two TaPIN proteins were significantly downregulated, and multiple auxin signalling genes were also differentially expressed after cold stress. We used RNAi technology to knock down the two TaPIN genes and the tiller number was significantly reduced in TaPINa-RNAi and TaPINb-RNAi wheat. Taken together, the results revealed that the ftin gene might directly or indirectly regulate multiple biological pathways to affect multiple phenotypic characteristics.
\end{abstract}

\section{Hosted file}

Spike development inhibition in the ftin mutant is associated with multiple phenotypic characteristics available at https://authorea.com/users/313917/articles/444324-spike-development-inhibitionin-the-ftin-mutant-is-associated-with-multiple-phenotypic-characteristics-and-regulated-multiplebiological-pathways 